\title{
Neurological and Neurosurgical Aspect of COVID-19 Pandemic
}

\author{
Aabishkar Bhattarai ${ }^{1}$, Bijaya Karki ${ }^{2}$, Nikunja Yogi ${ }^{3}$ \\ ${ }^{1}$ Department of Neurosurgery, Manipal College of Medical Sciences, Nepal \\ ${ }^{2}$ Department of Intensive Care Unit, Birat Medical College Teaching Hospital, Nepal \\ ${ }^{3}$ Department of Neurosurgery, Manipal College of Medical Sciences, Nepal
}

Correspondence:

Dr. Aabishkar Bhattarai

Medical Officer, Department of Neurosurgery

Manipal College of Medical Sciences, Nepal

Email: aabishkar.bhattarai@gmail.com

\begin{abstract}
Even though severe acute respiratory syndrome coronavirus 2 (SARS-CoV-2) (COVID-19) has been observed to mainly affect the pulmonary system, neurological (Central and Peripheral) involvements have already been reported in the literature. Also, it should be kept in mind that the patients may have COVID-19 regardless of the presence of respiratory symptoms, especially those presenting to the emergency departments (e.g. with road traffic accidents) or neurology outpatient clinics during the pandemic with neurological complaints and symptoms. A holistic approach is of great importance in protecting healthcare workers from infection as well as providing health-care to the patients and preventing late or misdiagnosis
\end{abstract}

$\mathrm{S}$ ince the emergence of the disease from Wuhan China in December 2019, the outbreak of acute severe respiratory failure syndrome (SARS) coronavirus 2 (SARS-CoV-2), commonly known as coronavirus disease (COVID$19)$, is rapidly spreading throughout the world. The World Health Organization declared COVID-19 a global pandemic as of March 11, 2020. ${ }^{1}$ Every country of the world is fighting to its best to contain the virus; however, the death toll has surpassed 777,218 as per 18 August $2020 .^{2}$ The COVID-19 infection has mostly shown to affect the respiratory, cardiovascular, and gastrointestinal system but the central nervous system (CNS) and peripheral nervous system (PNS) have not been spared too. Neurologists and neurosurgeons are also an integral part of a team of COVID-19 rescue plans working together with an emergency, intensive care, and respiratory physicians. And there has been a profound impact of COVID-19 on neurological clinics, surgeries, and neurological patients.

\section{Discussion:}

For patients with COVID-19, there is little definite knowledge about the neurological complications of the disease (e.g. risk factors, clinical features,
Pathophysiology, outcome, and prognosis). However, neurological symptomatology has been increasingly reported in recent publications. Neurological involvement in coronavirus disease 2019 (COVID-19) manifests under three situations: (a) neurological clinical features of viral infection, (b) complications after infection, and (c) COVID19 infection in patients with neurological comorbidity. ${ }^{3}$ More frequent neurological symptoms include headache (11-13\%), dizziness (8-17\%), altered level of consciousness $(8-9 \%)$, and hypogeusia, hyposmia, or anosmia (5\% ). ${ }^{4}$ On the other hand, hyposmia can be the unique symptom in carriers of COVID-19 which can also serve as a marker for identifying the otherwise asymptomatic but infected patients. Although the mechanism of invasion is not fully understood, COVID-19 appears to demonstrate neuroinvasive potential by using angiotensin-converting enzyme 2 (ACE-2) receptor to gain entry and infect both glial and neuronal cells that express ACE-2 receptors. ${ }^{5,6}$ Viral encephalitis with or without hemorrhagic necrosis involving mesial brain structures such as the mesial temporal lobes and thalamus have been documented. ${ }^{4}$

Neurological manifestations can be subdivided into the central nervous system (headache, dizziness, 
altered sensorium, seizures, ataxia, encephalitis, and stroke) and peripheral nervous system (skeletal muscle injury and peripheral nerve involvement including hyposmia, anosmia, hypogeusia, neuralgia) symptomatology. Post-infective neurological complications include demyelinating conditions like Guillain-Barre syndrome. ${ }^{3}$ Since the onset of the SARS-CoV-2 outbreak, one pulmonary manifestation that has received extensive focus with grave prognosis is Acute Respiratory Distress Syndrome (ARDS). It is suggested that a significant percentage of ARDS survivors may suffer long-term cognitive impairment. Acute injury to the blood-brain barrier, either in a scenario of mechanical ventilation in the majority of COVID-19 ARDS patients or due to the neurotropic potential of the virus has been implicated as the underlying mechanism for cognitive impairment following ARDS. As the pandemic continues to unfold, the number of people waning off mechanical ventilation will increase, and long-term cognitive outcomes may arise. It can be anticipated that there can be cognitive decline lasting for months in these post mechanically ventilated patients, but also some of them may progress to premature onset of dementia. ${ }^{3,6}$

While there is evidence suggesting that comorbidities such as hypertension, diabetes mellitus, increase the severity of COVID-19 illness, less is known on the risk for patients with neurological disorders. Although uncommon, the pre-existing Cerebrovascular disease may be a risk factor for poor outcomes in patients infected with COVID-19 patients. It is reported that a variety of patients with reduced mobility due to paresis or paralysis and those on immune-suppression therapy may be anticipated to be more susceptible to COVID-19infection. ${ }^{7}$

Traditionally, the neurological diagnostic and treatment approach has been face-to-face. COVID19 and mandatory social distancing have forced neurology practices worldwide to dramatically reshape their delivery of care. The practice of neurosurgery is yet susceptible to the havoc that COVID-19 has brought on. Neurology and neurosurgical practice patterns are being changed dramatically and constantly updated with publications demonstrating early experiences and recommendations based on facilities and regions most affected. However, to protect both patients and health-care professionals, alternative means of care are urgently needed. As healthcare resources had to be preserved for upcoming patients with COVID-19 and the in-hospital procedures possess increased risk of infection to uninfected patients and staff, neurosurgeons around the world have had to postpone non-emergent procedures. Teams of neurosurgeons have been re-deployed as an intensivist, neurosurgical patients have been rehoused in COVID-clean wards, protocols have been implemented to test patients ahead of neurosurgical procedures, and hospital systems have been reorganized to "hub-and-spoke" configurations to segregate neurosurgery to designated centers. On March 13, 2020, the American College of Surgeons (ACS) issued an unprecedented recommendation to minimize, postpone, or cancel electively scheduled operations. Non-urgent surgical procedures and medical appointments have been canceled to increase bed capacity and care for patients with the infection, while many neurologists have had to leave their clinics to help other physicians on the front-lines.

Similarly, neurosurgeons can readily determine that the conditions that can wait for the treatment until COVID-19 pandemics is thoroughly defeated and those conditions which require emergent treatment and surgeries. However, between these two extremes are the problematic cases, for which the risk of postponement is left to the unknowable pace of disease progression, or worse, dependent only on chance. Whereas there are many reasons to justify this postponement, according to the recommendation by the American College of Surgeons, the most important one is to preserve essential supplies, such as ventilators, for the avalanche of patients who will overwhelm resources in the predictable future. However, hospitals staffed by private-practice neurosurgeons are less likely than non-profit hospitals to mandate expansive cancelation of elective cases in the weeks following the ACS recommendation. And predictably, neurosurgeons working in the "forprofit" setting were also less likely to be in favor of broadly shutting down surgeries. As personal protective equipment ran scarce, the preservation of hospital staff quickly become egneuro, Volume 02, Issue 03, 2020 
another strong reason to defer non-emergent procedures. Taken together, postponing elective surgeries is not only a reasonable strategy, it seems to be the only rational path for neurosurgeons until the end of the pandemic is concerned that some operations may create ventilator-dependent patients without meaningful recovery, unfortunate always, but unjustifiable when resources are scarce during the pandemic. To care for the massive numbers of COVID patients, many hospital systems and surgeons are focused on conserving resources by limiting elective surgical procedures.

In this challenging period, neurologists and neurosurgeons, who are naturally primed and trained to respond and take on challenges in difficult situations, have the ideal opportunity to shine and make a true difference during this pandemic. In Nepal, many tertiary health centers have prepared duty rosters allowing limited members of the neuro-team to attend patients in the hospital in order to decrease exposure to virus by allowing efficient use of limited resources. ${ }^{8}$ Case discussions and updates on cases are later done on Internet-based applications like "Viber" and "Whatsapp." Consultations are being offered over the phone or via the Internet as reasonably as possible, focusing on prevention and general wellbeing. Emergency brain and spinal cord surgeries are being performed only in undeniable situations where there is an imminent threat to life or where delaying would make the condition permanently worse. $^{8}$

\section{Conclusion:}

To ensure optimum management of neurological patients, various researches and study of treatment algorithms will be essential for updating and adapting these approaches as the COVID-19 pandemic evolves. An important step will be to get a better understanding of the acute and postinfectious neurological manifestations of COVID19 to guide long-term management and health service reorganization. While it will take time to fully understand the neurological manifestations of COVID-19, collecting and sharing of data as well as a critical appraisal of the evidence will improve the care of neurological patients. Holistic knowledge of the spectrum of neurological consequences of COVID-19 is important to get a hold on the spread of the virus. With the gradual subsidence of the outbreak, it can be anticipated that several post infectious complications will surface up while rehabilitation measures will also deserve attention. Adequate caution has to be practiced while managing chronic neurology patients, particularly those requiring immunemodulator therapy since formulated guidelines are lacking at this point. Investigations of accompanying neurological involvement in patients admitted with severe respiratory tract infection and impaired consciousness are mandatory for correct management.

\section{References:}

1. "WHO Timeline - COVID-19."https://www.who.int/newsroom/detail/27-04-2020-who-timeline---covid-19 (accessed Jun. 24, 2020).

2. Worldometer, "Coronavirus Cases," Worldometer, 2020. https://www.worldometers.info/coronavirus/ (accessed Jul. $16,2020)$

3. D. Lahiri and A. Ardila, "COVID-19 Pandemic: A Neurological Perspective," Cureus, vol. 12, no. 4, Apr. 2020, doi: 10.7759/cureus.7889.

4. M. P. Wilson and A. S. Jack, "Coronavirus disease 2019 (COVID-19) in neurology and neurosurgery: A scoping review of the early literature," Clinical Neurology and Neurosurgery, vol. 193. Elsevier B.V., p. 105866, Jun. 01, 2020, doi: 10.1016/j.clineuro.2020.105866.

5. V. Montalvan, J. Lee, T. Bueso, J. De Toledo, and K. Rivas, "Neurological manifestations of COVID-19 and other coronavirus infections: A systematic review," Clinical Neurology and Neurosurgery, vol. 194. Elsevier B.V., p. 105921, Jul. 01, 2020, doi: 10.1016/j.clineuro.2020.105921.

6. D. Lahiri et al., "Neuroinvasive potential of a primary respiratory pathogen SARS- CoV2: Summarizing the evidences," Diabetes and Metabolic Syndrome: Clinical Research and Reviews, vol. 14, no. 5. Elsevier Ltd, pp. 1053-1060, Sep. 01, 2020, doi: 10.1016/j.dsx.2020.06.062.

7. The Lancet Neurology, "The neurological impact of COVID-19," Lancet Neurol., vol. 19, no. 6, p. 471, 2020, doi: 10.1016/S1474-4422(20)30142-3.

8. [A. Thapa, "COVID-19 and the Role of Neurosurgeons in Nepal," World Neurosurg., vol. 139, p. 629, Jul. 2020, doi: 10.1016/j.wneu.2020.05.093. 